\title{
Effect of some anti-inflammatory drugs on fibroblast-leucocyte interaction in vitro
}

\author{
M. YARON, I. YARON, AND D. ALLALOUF* \\ Rogoff-Wellcome Medical Research Institute, Beilinson Hospital, and Tel-Aviv University Medical School, \\ Petah Tikva, Israel
}

Inflammation of an affected joint is accompanied by a local overproduction of hyaluronic acid. Artificially induced inflammation in the experimental animal is also accompanied by a local overproduction of acid mucopolysaccharides (Delaunay and Basin, 1965). Previous studies have shown that addition of inflammatory cells to cultured human synovial fibroblasts induce an increase of hyaluronic acid production along with an increased glucose uptake by the fibroblasts (Yaron and Castor, 1969). The present study on the effect of anti-inflammatory drugs on the metabolic changes of the connective tissue cell caused by leucocyte material might contribute to a better understanding of the effect of antiinflammatory drugs in vivo.

\section{Methods and material}

The drugs used in our experiments were:

(1) Hydrocortisone acetate (Fredriksberg Chemical Laboratories Ltd., Copenhagen).

(2) Hydrocortisone Na-succinate (Hydro-adreson. Organon, Holland).

(3) Na-salicylate (Dr. E. Fresenius, Germany).

(4) Indomethacin (Indocin. Merck Sharp \& Dohme, USA).

(5) Phenylbutazone (Butazolidin. Geigy, Switzerland).

(6) Oxyphenbutazone (Tanderil. Geigy, Switzerland).

Fibroblast cultures derived from human synovial tissue obtained in the operation room were grown as monolayers in tissue culture flasks as described by Castor, Prince, and Dorstewitz (1962). Cells were nourished by a semisynthetic medium consisting of 80 per cent. medium 1066, 10 per cent. foetal calf serum, and 10 per cent. heat-inactivated human serum, supplemented with L-glutamine, penicillin, and streptomycin.

In experiments 1 and 2, Parker medium 199 was used instead of medium 1066.

Fibroblasts were planted in duplicate or triplicate cultures in T-30 flasks containing $5 \mathrm{ml}$. medium.

Strains PL and AL were derived from patients with classical rheumatoid arthritis at the time of synovectomy of the knee joint. All the other strains were derived from synovial tissue obtained at meniscus tear operations from otherwise healthy persons.

Leucocytes were obtained from heparinzed peripheral blood (50 units heparin $/ \mathrm{ml}$. blood) of healthy medical personnel or patients taking no drugs or very few drugs (no anti-inflammatory drugs). After red blood cells sedimentation, the suspension of leucocytes in plasma was centrifuged at $200 \mathrm{G}$. for $10 \mathrm{~min}$.; the leucocytes were then washed once with medium, disrupted by three cycles of freezing and thawing, and added to the fibroblasts in the tissue culture flasks, $10 \times 10^{6}$ frozen thawed leucocytes per flask $(5 \mathrm{ml}$. medium), along with complete medium replacement. At the time of leucocyte addition, 0.1 or $0.2 \mathrm{ml}$. of a solution or a suspension of the antiinflammatory drug in saline or in medium 199 were added to some of the flasks. The 'interaction' period was the last 2 days or the last day of a 7-day growth cycle. At the end of this period the medium was removed and the fibroblasts trypsinized and counted. Hyaluronic acid in the culture media was measured by a method described by Castor, Wright, and Buckingham (1968) whereby the mucopolysaccharide is isolated and its uronic acid content determined. It has already been shown (Yaron and Castor, 1969) that the mucopolysaccharide produced by synovial fibroblasts in tissue culture is indeed hyaluronic acid.

Glucose consumption was determined by estimating the residual glucose in culture media with a glucose oxidase method (Kingsley and Getchell, 1960).

\section{Results}

Each Table in the following text represents a separate experiment, and the detailed format of individual experiments can be best appreciated from these Tables (I to IX).

The addition of frozen thawed leucocytes to cultured monolayers of human synovial fibroblasts caused a 23 to 140 per cent. increase of hyaluronic acid synthesis (expressed per fibroblast) and a 5 to 100 per cent. increase of glucose consumption (per flask) after 1 or 2 days 'interaction' time. This stimulation was inhibited by adding anti-inflammatory drugs with the leucocytes: 
Hydrocortisone acetate $1 \mu \mathrm{g} . / \mathrm{ml}$. (40 and 100 per cent. inhibition of hyaluronate overproduction in two experiments: Tables I, II);

Hydrocortisone Na-succinate 1 and $10 \mu \mathrm{g} . / \mathrm{ml}$. (100 per cent. in three experiments: Tables III, IV, IX);

Indomethacin 2 or $4 \mu \mathrm{g} . / \mathrm{ml}$. (100 per cent. in three experiments: Tables VII, VIII, IX);

Phenylbutazone 10 or $20 \mu \mathrm{g} / \mathrm{ml}$. (75 and 100 per cent. respectively: Tables VIII, IX);

Oxyphenbutazone $10 \mu \mathrm{g} . / \mathrm{ml}$. (74 per cent.: Table IX);

Sodium salicylate $200 \mu \mathrm{g} . / \mathrm{ml}$. (88 and 44 per cent.; Tables V, VI).

The inhibition of hyaluronate overproduction was associated with a 5 to 47 per cent. decrease of glucose uptake (per flask).

The addition of hydrocortisone, indomethacin, phenylbutazone, and oxyphenbutazone without leucocytes caused a reduction in the hyaluronate output and glucose uptake of the fibroblasts. This effect was not observed when sodium salicylate was used, although this drug did inhibit the overproduction of hyaluronate induced by leucocytes (Tables V and VI). Leucocyte material or antiinflammatory drugs, when added alone, caused in some experiments a slight decrease in the final fibroblast count and in some a slight increase, while in the rest it had no significant effect.

The addition of anti-inflammatory drugs together with leucocytes had no significant effect on fibroblast proliferation: in only one experiment (Table IX) the addition of indomethacin and leucocytes together seemed to stimulate fibroblast proliferation.
There was no noticeable toxic eftect on the fibroblasts with any of the additives used. The mucopolysaccharide content in the amounts of frozen-thawed leucocytes used was found to be negligible.

\section{Discussion}

All the anti-inflammatory drugs used had an inhibitory effect on the overproduction of hyaluronic acid and increased glucose consumption of the cultured fibroblasts induced by addition of frozenthawed leucocytes. The drug concentrations used in our experiments are considered to be pharmacological levels in vivo. The use of dead leucocytes was preferred in order to exclude possible biochemical changes caused by the metabolism of living leucocytes. It has already been shown (Yaron and Castor, 1969) that the addition of either live or dead leucocytes to fibroblast cultures induce similar changes in the fibroblast metabolism. Kalbhen, Karzel, and Domenjoz (1967) showed that indomethacin, oxyphenbutazone, Na-salicylate and mefenamic acid inhibit the rate of glucosamine- $\mathrm{C}^{14}$ incorporation into the mucopolysaccharides produced by fibroblasts in vitro. Castor (1962) has shown that adrenocorticoids suppress mucopolysaccharide formation of cultured synovial fibroblasts. Castor, Dorstewitz, Wright and Ritchie (1969) have also described the inhibitory effect of hydrocortisone, sodium fluoride, and sodium salicylate on the overproduction of hyaluronic synthesis of cultured synovial fibroblasts after the addition of frozen-thawed fibroblasts.

$A$ reduction of the inflammatory process in the

Table I Effect of hydrocortisone acetate on fibroblast-leucocyte interaction

\begin{tabular}{|c|c|c|c|c|c|c|}
\hline $\begin{array}{l}\text { Fibroblast } \\
\text { strain }^{a}\end{array}$ & Additive & $\begin{array}{l}\text { Final cell } \\
\text { count flask } \\
\times 10^{6}\end{array}$ & $\begin{array}{l}\text { Residual } \\
\text { medium } \\
\text { glucose } \\
(\mathrm{mg} . / 100 \mathrm{ml} .)\end{array}$ & $\begin{array}{l}\text { Glucose } \\
\text { uptake } \\
\text { (mg.|flask } \\
\text { per } 2 \text { days) }\end{array}$ & $\begin{array}{l}\text { Total } \\
\text { HA|flask } \\
(\mu g .)\end{array}$ & $\begin{array}{l}\text { HA } \\
\text { synthesis rate } \\
\text { (pg./cell per day) }\end{array}$ \\
\hline SB-H & None & $\begin{array}{l}1.0 \\
(0.9 \text { to } 1.05)\end{array}$ & $\begin{array}{l}45 \\
(45 \text { to } 45)\end{array}$ & $2 \cdot 45$ & $\begin{array}{l}54 \cdot 0 \\
(51 \cdot 7 \text { to } 58 \cdot 6)\end{array}$ & $\begin{array}{l}25 \cdot 5 \\
(24 \cdot 6 \text { to } 28 \cdot 7)\end{array}$ \\
\hline SB-H & 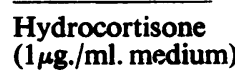 & $\begin{array}{l}0.9 \\
(0.8 \text { to } 1.0)\end{array}$ & $\begin{array}{l}54 \cdot 7 \\
(54 \text { to } 56)\end{array}$ & 1.96 & $\begin{array}{l}27 \cdot 6 \\
(25 \cdot 3 \text { to } 32 \cdot 2)\end{array}$ & $\begin{array}{l}15 \cdot 3 \\
(14 \cdot 0 \text { to } 16 \cdot 1)\end{array}$ \\
\hline$\overline{\mathrm{SB}-\mathrm{H}}$ & Leucocytes & $\begin{array}{l}0.85 \\
(0.85 \text { to } 0.85)\end{array}$ & $\begin{array}{l}26 \cdot 0 \\
\text { (25 to 27) }\end{array}$ & $3 \cdot 40$ & $\begin{array}{l}100 \cdot 3 \\
(96 \cdot 5 \text { to } 108 \cdot 0)\end{array}$ & $\begin{array}{l}57 \cdot 1 \\
(56 \cdot 7 \text { to } 57 \cdot 6)\end{array}$ \\
\hline$\overline{\text { SB-H }}$ & 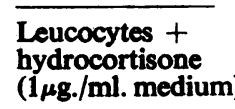 & $\begin{array}{l}0.92 \\
(0.92 \text { to } 0.92)\end{array}$ & $\begin{array}{l}44 \\
(40 \text { to } 46)\end{array}$ & $\overline{2 \cdot 50}$ & $\begin{array}{l}46 \cdot 0 \\
(46 \cdot 0 \text { to } 46 \cdot 0)\end{array}$ & $\begin{array}{l}25 \cdot 0 \\
(25 \cdot 0 \text { to } 25 \cdot 0)\end{array}$ \\
\hline
\end{tabular}

a SB refers to initials of donor, and $H$ indicates the number of times (8) the strain has been subcultured

$b$ Fibroblast inoculum $=0.6 \times 10^{\circ}$ flask. Individual values are the mean of duplicate or triplicate determinations; figures in parentheses indicate

c the absolute range of the data

c Glucose concentration in initial medium $=94 \mathrm{mg} . / 100 \mathrm{ml}$.

Leucocytes add
growth cycle

growth cycle
$\mathbf{H A}=$ hyaluronic acid 
Table II Effect of hydrocortisone acetate on fibroblast-leucocyte interaction ${ }^{a}$

\begin{tabular}{|c|c|c|c|c|c|c|}
\hline $\begin{array}{l}\text { Fibroblast } \\
\text { strain }\end{array}$ & Additive & $\begin{array}{l}\text { Final cell } \\
\text { count/flask } \\
\times 10^{8}\end{array}$ & $\begin{array}{l}\text { Residual } \\
\text { medium } \\
\text { glucose }^{b} \\
(\mathrm{mg} . / 100 \mathrm{ml} .)\end{array}$ & $\begin{array}{l}\text { Glucose } \\
\text { uptake } \\
\text { (mg.|flask } \\
\text { per } 2 \text { days) }\end{array}$ & $\begin{array}{l}\text { Total } \\
\text { HA/flask } \\
(\mu g .)\end{array}$ & $\begin{array}{l}\text { HA } \\
\text { synthesis rate } \\
\text { (pg./cell per day) }\end{array}$ \\
\hline RY-D & None & $\begin{array}{l}1 \cdot 14 \\
(1 \cdot 12 \text { to } 1 \cdot 17)\end{array}$ & $\begin{array}{l}66 \cdot 5 \\
(64 \text { to 69) }\end{array}$ & $1 \cdot 92$ & $\begin{array}{l}54 \cdot 0 \\
(54 \cdot 0 \text { to } 54 \cdot 0)\end{array}$ & $\begin{array}{l}23 \cdot 8 \\
(23 \cdot 5 \text { to } 24 \cdot 1)\end{array}$ \\
\hline RY-D & Leucocytes & $\begin{array}{l}1 \cdot 46 \\
(1 \cdot 40 \text { to } 1 \cdot 52)\end{array}$ & $\begin{array}{l}29 \\
(27 \text { to } 31)\end{array}$ & $3 \cdot 80$ & $\begin{array}{l}166 \cdot 6 \\
(166 \cdot 6 \text { to } 166 \cdot 6)\end{array}$ & $\begin{array}{l}57 \cdot 1 \\
(54 \cdot 8 \text { to } 59 \cdot 5)\end{array}$ \\
\hline RY-D & $\begin{array}{l}\text { Leucocytes }+ \\
\text { hydrocortisone } \\
\text { (1 } \mu \mathrm{g} . / \mathrm{ml} . \text { medium) }\end{array}$ & $\begin{array}{l}1 \cdot 30 \\
\text { No duplicate }\end{array}$ & 52 & $2 \cdot 65$ & $114 \cdot 8$ & $44 \cdot 1$ \\
\hline
\end{tabular}

a Number of fibroblasts inoculated, leucocytes added, and interaction time, as in Table I

$b$ Glucose concentration in initial medium $=105 \mathrm{mg} . / 100 \mathrm{ml}$.

Table III Effect of hydrocortisone Na-succinate on fibroblast-leucocyte interaction

\begin{tabular}{|c|c|c|c|c|c|c|}
\hline $\begin{array}{l}\text { Fibroblast } \\
\text { strain }\end{array}$ & Additive & $\begin{array}{l}\text { Final cell } \\
\text { count/flask } \\
\times 10^{6}\end{array}$ & $\begin{array}{l}\text { Residual } \\
\text { medium } \\
\text { glucose } \\
(\mathrm{mg} .100 \mathrm{ml} .)\end{array}$ & $\begin{array}{l}\text { Glucose } \\
\text { uptake } \\
\text { (mg./flask } \\
\text { per } 2 \text { days) }\end{array}$ & $\begin{array}{l}\text { Total } \\
\text { HA/flask } \\
(\mu g .)\end{array}$ & $\begin{array}{l}\text { HA } \\
\text { synthesis rate } \\
\text { (pg./cell per day) }\end{array}$ \\
\hline PL-E & None & $\begin{array}{l}2 \cdot 3 \\
(2 \cdot 2 \text { to } 2 \cdot 4)\end{array}$ & $\begin{array}{l}8 \cdot 6 \\
(8 \cdot 2 \text { to } 9 \cdot 0)\end{array}$ & $3 \cdot 7$ & $\begin{array}{l}116 \\
(106 \text { to } 126)\end{array}$ & $\begin{array}{l}25 \cdot 1 \\
(24 \cdot 1 \text { to } 26 \cdot 2)\end{array}$ \\
\hline PL-E & Leucocytes $^{c}$ & $\begin{array}{l}2 \cdot 4 \\
(2 \cdot 4 \text { to } 2 \cdot 4)\end{array}$ & $\begin{array}{l}5 \cdot 7 \\
(4 \cdot 8 \text { to } 6 \cdot 6)\end{array}$ & $3 \cdot 9$ & $\begin{array}{l}153 \\
(140 \text { to } 166)\end{array}$ & $\begin{array}{l}31 \cdot 8 \\
(29 \cdot 0 \text { to } 34 \cdot 6)\end{array}$ \\
\hline PL-E & $\begin{array}{l}\text { Hydrocortisone } \\
\text { (1 } \mu \mathrm{g} . / \mathrm{ml} . \text { medium })\end{array}$ & $\begin{array}{l}1 \cdot 6 \\
(1 \cdot 6 \text { to } 1 \cdot 6)\end{array}$ & $\begin{array}{l}34 \cdot 3 \\
(33 \cdot 8 \text { to } 34 \cdot 8)\end{array}$ & $2 \cdot 4$ & $\begin{array}{l}43 \cdot 6 \\
(36 \cdot 6 \text { to } 50 \cdot 7)\end{array}$ & $\begin{array}{l}13 \cdot 6 \\
(11 \cdot 4 \text { to } 15 \cdot 8)\end{array}$ \\
\hline PL-E & $\begin{array}{l}\text { Leucocytes }+ \\
\text { hydrocortisone } \\
(1 \mu \mathrm{g} . / \mathrm{ml} . \text { medium })\end{array}$ & $\begin{array}{l}2 \cdot 0 \\
(2 \cdot 0 \text { to } 2 \cdot 0)\end{array}$ & $\begin{array}{l}24 \\
(22 \text { to } 26)\end{array}$ & $3 \cdot 0$ & $\begin{array}{l}49 \cdot 5 \\
(49 \cdot 5 \text { to } 49 \cdot 5)\end{array}$ & $\begin{array}{l}12 \cdot 4 \\
(12 \cdot 4 \text { to } 12 \cdot 4)\end{array}$ \\
\hline
\end{tabular}

$a$ Fibroblast inoculum $=0.72 \times 10^{\circ}$ per flask

b Glucose concentration in initial medium $=83.4 \mathrm{mg} / 100 \mathrm{ml}$.

c Leucocytes added (frozen-thawed): $5 \times 10^{\circ}$ per flask. 'Interaction' period 2 days

Table IV Effect of hydrocortisone Na-succinate on fibroblast-leucocyte interactiona

\begin{tabular}{|c|c|c|c|c|c|c|}
\hline $\begin{array}{l}\text { Fibroblast } \\
\text { strain }\end{array}$ & Additive & $\begin{array}{l}\text { Final cell } \\
\text { count/fiask } \\
\times 10^{8}\end{array}$ & $\begin{array}{l}\text { Residual } \\
\text { medium } \\
\text { glucose } \\
(\mathrm{mg} . / 100 \mathrm{ml} .)\end{array}$ & $\begin{array}{l}\text { Glucose } \\
\text { uptake } \\
\text { (mg./flask } \\
\text { per } 2 \text { days) }\end{array}$ & $\begin{array}{l}\text { Total } \\
\text { HA/flask } \\
(\mu g .)\end{array}$ & $\begin{array}{l}\text { HA } \\
\text { synthesis rate } \\
\text { (pg./cell per day) }\end{array}$ \\
\hline GO-E & None & $\begin{array}{l}1 \cdot 43 \\
(1 \cdot 40 \text { to } 1 \cdot 46)\end{array}$ & $\begin{array}{l}43 \cdot 5 \\
(40 \cdot 3 \text { to } 46 \cdot 9)\end{array}$ & $2 \cdot 0$ & $\begin{array}{l}77 \cdot 8 \\
(77 \cdot 8 \text { to } 77 \cdot 8)\end{array}$ & $\begin{array}{l}27 \cdot 2 \\
(26 \cdot 6 \text { to } 27 \cdot 8)\end{array}$ \\
\hline GO-E & Leucocytes & $\begin{array}{l}1 \cdot 28 \\
(1 \cdot 28 \text { to } 1 \cdot 28)\end{array}$ & $\begin{array}{l}32 \cdot 7 \\
(31 \cdot 8 \text { to } 33 \cdot 6)\end{array}$ & $2 \cdot 5$ & $\begin{array}{l}142 \\
\text { (126 to } 158)\end{array}$ & $\begin{array}{l}55 \cdot 4 \\
(49 \cdot 2 \text { to } 61 \cdot 7)\end{array}$ \\
\hline GO-E & $\begin{array}{l}\text { Hydrocortisone } \\
(1 \mu \mathrm{g} / \mathrm{ml} \text {. medium) }\end{array}$ & $\begin{array}{l}1 \cdot 4 \\
(1 \cdot 4 \text { to } 1 \cdot 4)\end{array}$ & $\begin{array}{l}54 \cdot 6 \\
(51 \cdot 7 \text { to } 57 \cdot 5)\end{array}$ & $1 \cdot 45$ & $\begin{array}{l}57 \cdot 7 \\
(57 \cdot 7 \text { to } 57 \cdot 7)\end{array}$ & $\begin{array}{l}20 \cdot 6 \\
(20 \cdot 6 \text { to } 20 \cdot 6)\end{array}$ \\
\hline GO-E & $\begin{array}{l}\text { Leucocytes t } \\
\text { hydrocortisone } \\
\text { (1 } \mu \mathrm{g} . / \mathrm{ml} \text {. medium) }\end{array}$ & $\begin{array}{l}1 \cdot 4 \\
(1 \cdot 4 \text { to } 1 \cdot 4)\end{array}$ & $\begin{array}{l}53 \cdot 0 \\
(51 \cdot 7 \text { to } 54 \cdot 0)\end{array}$ & $1 \cdot 5$ & $\begin{array}{l}67 \cdot 2 \\
(61 \cdot 3 \text { to } 73 \cdot 1)\end{array}$ & $\begin{array}{l}24 \cdot 0 \\
(21 \cdot 9 \text { to } 26 \cdot 1)\end{array}$ \\
\hline
\end{tabular}

Number of fibroblasts inoculated, leucocytes added, interaction time, and glucose concentration in initial medium, as in Table III 
Table V Effect of sodium salicylate on fibroblast-leucocyte interaction

\begin{tabular}{|c|c|c|c|c|c|c|}
\hline $\begin{array}{l}\text { Fibroblast } \\
\text { strain }\end{array}$ & Additive & $\begin{array}{l}\text { Final cell } \\
\text { count/flask } \\
\times 10^{6}\end{array}$ & $\begin{array}{l}\text { Residual } \\
\text { medium } \\
\text { glucose }^{b} \\
(\mathrm{mg} . / 100 \mathrm{ml} .)\end{array}$ & $\begin{array}{l}\text { Glucose } \\
\text { uptake } \\
\text { (mg./flask } \\
\text { per } 2 \text { days) }\end{array}$ & $\begin{array}{l}\text { Total } \\
\text { HAlflask } \\
(\mu g .)\end{array}$ & $\begin{array}{l}\text { HA } \\
\text { synthesis rate } \\
\text { (pg./cell per day) }\end{array}$ \\
\hline OA-D & None & $\begin{array}{l}1 \cdot 0 \\
(1 \cdot 0 \text { to } 1 \cdot 0)\end{array}$ & $\begin{array}{l}83 \\
(82 \text { to } 84)\end{array}$ & $1 \cdot 1$ & $\begin{array}{l}41 \cdot 5 \\
(41 \text { to } 42)\end{array}$ & $\begin{array}{l}20 \cdot 7 \\
(20 \cdot 5 \text { to } 21 \cdot 0)\end{array}$ \\
\hline OA-D & Leucocytes $^{c}$ & $\begin{array}{l}0.82 \\
(0.82 \text { to } 0.85)\end{array}$ & $\begin{array}{l}71 \cdot 5 \\
(66 \text { to } 77)\end{array}$ & $1 \cdot 7$ & $\begin{array}{l}57 \cdot 5 \\
(55 \text { to } 60)\end{array}$ & $\begin{array}{l}34 \cdot 8 \\
(34 \cdot 4 \text { to } 35 \cdot 3)\end{array}$ \\
\hline OA-D & $\begin{array}{l}\text { Na salicylate } \\
(200 \mu \mathrm{g} . / \mathrm{ml} . \\
\text { medium })\end{array}$ & $\begin{array}{l}0.92 \\
(0.90 \text { to } 0.95)\end{array}$ & $\begin{array}{l}90 \\
(88 \text { to } 92)\end{array}$ & 0.8 & $\begin{array}{l}40 \cdot 0 \\
(40 \cdot 0 \text { to } 40 \cdot 0)\end{array}$ & $\begin{array}{l}21 \cdot 1 \\
(20 \cdot 1 \text { to } 22 \cdot 2)\end{array}$ \\
\hline OA-D & $\begin{array}{l}\text { Leucocytes }+ \\
\text { Na salicylate } \\
(200 \mu \mathrm{g} . / \mathrm{ml} \text {. } \\
\text { medium) }\end{array}$ & $\begin{array}{l}0.90 \\
(0.85 \text { to } 0.95)\end{array}$ & $\begin{array}{l}87 \cdot 5 \\
(86 \text { to } 89)\end{array}$ & 0.9 & $\begin{array}{l}41 \cdot 5 \\
(39 \text { to } 42)\end{array}$ & $\begin{array}{l}22 \cdot 5 \\
(22 \cdot 1 \text { to } 22 \cdot 9)\end{array}$ \\
\hline
\end{tabular}

$a$ Fibroblast inoculum $=0.45 \times 10^{\circ}$ per flask $b$ Glucose concentration in initial medium $=106 \mathrm{mg} .100 \mathrm{ml}$.

$c$ Leucocytes added (frozen-thawed): $10 \times 10^{\circ}$ per flask. 'Interaction' period 2 days

Table VI Effect of sodium salicylate on fibroblast-leucocyte interaction

\begin{tabular}{|c|c|c|c|c|c|c|}
\hline $\begin{array}{l}\text { Fibroblast } \\
\text { strain }\end{array}$ & Additive & $\begin{array}{l}\text { Final cell } \\
\text { count flask } \\
\times 10^{6}\end{array}$ & $\begin{array}{l}\text { Residual } \\
\text { medium } \\
\text { glucose }^{b} \\
(\mathrm{mg} . / 100 \mathrm{ml} .)\end{array}$ & $\begin{array}{l}\text { Glucose } \\
\text { uptake } \\
\text { (mg.|flask } \\
\text { per } 2 \text { days) }\end{array}$ & $\begin{array}{l}\text { Total } \\
\text { HA/flask } \\
(\mu g .)\end{array}$ & $\begin{array}{l}\text { HA } \\
\text { synthesis rate } \\
\text { (pg./cell per day) }\end{array}$ \\
\hline MN-D & None & $\begin{array}{l}2 \cdot 17 \\
(2 \cdot 11 \text { to } 2 \cdot 23)\end{array}$ & $\begin{array}{l}56 \\
(54 \text { to } 58)\end{array}$ & $1 \cdot 9$ & $\begin{array}{l}50 \cdot 9 \\
(50 \cdot 4 \text { to } 51 \cdot 4)\end{array}$ & $\begin{array}{l}23 \cdot 5 \\
(22 \cdot 6 \text { to } 24 \cdot 4)\end{array}$ \\
\hline MN-D & Leucocytes $^{c}$ & $\begin{array}{l}1.93 \\
(1.91 \text { to } 1.96) \\
\end{array}$ & $\begin{array}{l}43 \cdot 5 \\
(43 \text { to } 44) \\
\end{array}$ & $2 \cdot 6$ & $\begin{array}{l}76 \cdot 0 \\
(73 \cdot 0 \text { to } 79 \cdot 0)\end{array}$ & $\begin{array}{l}39 \cdot 3 \\
(37 \cdot 2 \text { to } 41 \cdot 4) \text { 흔 }\end{array}$ \\
\hline MN-D & $\begin{array}{l}\text { Na salicylate } \\
(200 \mu \mathrm{g} . / \mathrm{ml} . \\
\text { medium })\end{array}$ & $\begin{array}{l}1.96 \\
(1.93 \text { to } 2 \cdot 0)\end{array}$ & $\begin{array}{l}54 \cdot 5 \\
(54 \text { to } 55)\end{array}$ & $2 \cdot 0$ & $\begin{array}{l}47 \cdot 1 \\
(45 \cdot 7 \text { to } 48 \cdot 5)\end{array}$ & $\begin{array}{l}24 \cdot 0 \\
(23 \cdot 7 \text { to } 24 \cdot 3)\end{array}$ \\
\hline MN-D & $\begin{array}{l}\text { Leucocytes }+ \\
\mathrm{Na} \text { salicylate } \\
(200 \mu \mathrm{g} . / \mathrm{ml} \text {. } \\
\text { medium })\end{array}$ & $\begin{array}{l}2 \cdot 09 \\
(2 \cdot 0 \text { to } 2 \cdot 26)\end{array}$ & $\begin{array}{l}51 \cdot 7 \\
(50 \text { to } 53)\end{array}$ & $2 \cdot 2$ & $\begin{array}{l}66 \cdot 4 \\
(63 \cdot 8 \text { to } 68 \cdot 5)\end{array}$ & $\begin{array}{l}32 \cdot 0 \\
(30 \cdot 0 \text { to } 33 \cdot 8)\end{array}$ \\
\hline
\end{tabular}

$a$ Fibroblast inoculum $=0.5 \times 10^{6}$ per flask $\quad b$ Glucose concentration in initial medium $=95 \mathrm{mg} . / 100 \mathrm{ml}$.

$c$ Leucocytes added (frozen-thawed): $10 \times 10^{\circ}$ per flask. 'Interaction' period for the two kinds of cells together was the last day of a 7-day growth cycle

Table VII Effect of indomethacin on fibroblast-leucocyte interaction

\begin{tabular}{|c|c|c|c|c|c|c|}
\hline $\begin{array}{l}\text { Fibroblast } \\
\text { strain }\end{array}$ & Additive & $\begin{array}{l}\text { Final cell } \\
\text { count/flask } \\
\times 10^{6}\end{array}$ & $\begin{array}{l}\text { Residual } \\
\text { medium } \\
\text { glucose } \\
(\mathrm{mg} . / 100 \mathrm{ml} .)\end{array}$ & $\begin{array}{l}\text { Glucose } \\
\text { uptake } \\
\text { (mg.|flask } \\
\text { per } 2 \text { days) }\end{array}$ & $\begin{array}{l}\text { Total } \\
\text { HAlflask } \\
(\mu g .)\end{array}$ & $\begin{array}{l}\text { HA } \\
\text { synthesis rate } \\
\text { (pg./cell per day) }\end{array}$ \\
\hline AL-D & None & $\begin{array}{l}0.89 \\
(0.88 \text { to } 0.90)\end{array}$ & $\begin{array}{l}49 \\
(49 \text { to } 49)\end{array}$ & $1 \cdot 3$ & $\begin{array}{l}38 \cdot 5 \\
(34 \text { to } 43)\end{array}$ & $\begin{array}{l}21 \cdot 6 \\
(19 \cdot 4 \text { to } 23 \cdot 8)\end{array}$ \\
\hline$\overline{\mathrm{AL}-\mathrm{D}}$ & Leucocytes $^{c}$ & $\begin{array}{l}0.95 \\
(0.95 \text { to } 0.95)\end{array}$ & $\begin{array}{l}38 \cdot 5 \\
(37 \text { to } 40)\end{array}$ & $1 \cdot 9$ & $\begin{array}{l}51 \\
(51 \text { to } 51)\end{array}$ & $\begin{array}{l}26 \cdot 7 \\
(26 \cdot 7 \text { to } 26 \cdot 7)\end{array}$ \\
\hline AL-D & $\begin{array}{l}\text { Indomethacin } \\
(2 \mu \mathrm{g} . / \mathrm{ml} \text {. medium })\end{array}$ & $\begin{array}{l}1 \cdot 17 \\
(1 \cdot 17 \text { to } 1 \cdot 17)\end{array}$ & $\begin{array}{l}53 \\
(52 \text { to } 54)\end{array}$ & $1 \cdot 1$ & $\begin{array}{l}24 \\
\text { (24 to } 24)\end{array}$ & $\begin{array}{l}10 \cdot 2 \\
(10 \cdot 2 \text { to } 10 \cdot 2)\end{array}$ \\
\hline AL-D & $\begin{array}{l}\text { Leucocytes }+ \\
\text { indomethacin } \\
(2 \mu \mathrm{g} . / \mathrm{ml} \text {. medium })\end{array}$ & $\begin{array}{l}1 \cdot 0 \\
(1 \cdot 0 \text { to } 1 \cdot 0)\end{array}$ & $\begin{array}{l}41 \cdot 5 \\
(41 \text { to } 42)\end{array}$ & $1 \cdot 7$ & $\begin{array}{l}40 \cdot 5 \\
(39 \text { to } 42)\end{array}$ & $\begin{array}{l}20 \cdot 2 \\
(19 \cdot 5 \text { to } 21 \cdot 0)\end{array}$ \\
\hline
\end{tabular}


Table VIII Effect of indomethacin and phenylbutazone on fibroblast-leucocyte interaction

\begin{tabular}{|c|c|c|c|c|c|c|}
\hline $\begin{array}{l}\text { Fibroblast } \\
\text { strain }\end{array}$ & Additive & $\begin{array}{l}\text { Final cell } \\
\text { count/flask } \\
\times 10^{\circ}\end{array}$ & $\begin{array}{l}\text { Residual } \\
\text { medium } \\
\text { glucose } \\
(\mathrm{mg} . / 100 \mathrm{ml} .)\end{array}$ & $\begin{array}{l}\text { Glucose } \\
\text { uptake } \\
\text { (mg.flask } \\
\text { per } 2 \text { days) }\end{array}$ & $\begin{array}{l}\text { Total } \\
\text { HAlflask } \\
(\mu g .)\end{array}$ & $\begin{array}{l}\text { HA } \\
\text { synthesis rate } \\
\text { (pg./cell per day) }\end{array}$ \\
\hline VS-D & None & $\begin{array}{l}2 \cdot 36 \\
(2 \cdot 22 \text { to } 2 \cdot 49)\end{array}$ & $\begin{array}{l}63 \cdot 3 \\
(63 \text { to } 64)\end{array}$ & $1 \cdot 0$ & $\begin{array}{l}87 \cdot 1 \\
(85 \cdot 2 \text { to } 90 \cdot 9)\end{array}$ & $\begin{array}{l}18 \cdot 4 \\
(17 \cdot 1 \text { to } 19 \cdot 3)\end{array}$ \\
\hline VS-D & Leucocytes $^{c}$ & $\begin{array}{l}2 \cdot 09 \\
(2 \cdot 0 \text { to } 2 \cdot 24)\end{array}$ & $\begin{array}{l}42 \\
(38 \text { to } 44)\end{array}$ & $2 \cdot 0$ & $\begin{array}{l}112 \cdot 0 \\
(101 \cdot 1 \text { to } 127 \cdot 0)\end{array}$ & $\begin{array}{l}26 \cdot 6 \\
(24 \cdot 7 \text { to } 28 \cdot 3)\end{array}$ \\
\hline VS-D & $\begin{array}{l}\text { Indomethacin } \\
\text { (4 } \mu \mathrm{g} . / \mathrm{ml} \text {. medium) }\end{array}$ & $\begin{array}{l}2 \cdot 26 \\
(2 \cdot 20 \text { to } 2 \cdot 33)\end{array}$ & $\begin{array}{l}67 \\
(66 \text { to } 68)\end{array}$ & 0.8 & $\begin{array}{l}58 \cdot 0 \\
(54 \cdot 6 \text { to } 61 \cdot 4)\end{array}$ & $\begin{array}{l}12 \cdot 8 \\
(12 \cdot 4 \text { to } 13 \cdot 2)\end{array}$ \\
\hline VS-D & $\begin{array}{l}\text { Leucocytes }+ \\
\text { indomethacin } \\
(4 \mu \mathrm{g} . / \mathrm{ml} \text {. medium })\end{array}$ & $\begin{array}{l}2 \cdot 54 \\
(2 \cdot 38 \text { to } 2 \cdot 70)\end{array}$ & $\begin{array}{l}53 \cdot 5 \\
(53 \text { to } 54)\end{array}$ & $1 \cdot 5$ & $\begin{array}{l}92 \cdot 1 \\
(85 \cdot 2 \text { to } 98 \cdot 9)\end{array}$ & $\begin{array}{l}18 \cdot 1 \\
(17 \cdot 9 \text { to } 18 \cdot 3)\end{array}$ \\
\hline VS-D & $\begin{array}{l}\text { Phenylbutazone } \\
\text { (20 } \mu \mathrm{g} . / \text { ml. medium) }\end{array}$ & $\begin{array}{l}2 \cdot 41 \\
(2 \cdot 28 \text { to } 2 \cdot 55)\end{array}$ & $\begin{array}{l}68 \\
(68 \text { to } 68)\end{array}$ & 0.75 & $\begin{array}{l}60 \cdot 2 \\
(59 \cdot 1 \text { to } 61 \cdot 4)\end{array}$ & $\begin{array}{l}12 \cdot 5 \\
(12 \cdot 0 \text { to } 13 \cdot 0)\end{array}$ \\
\hline VS-D & $\begin{array}{l}\text { Leucocytes }+ \\
\text { phenylbutazone } \\
(20 \mu \mathrm{g} . / \mathrm{ml} \text {. medium })\end{array}$ & $\begin{array}{l}2 \cdot 23 \\
(2 \cdot 0 \text { to } 2 \cdot 47)\end{array}$ & $\begin{array}{l}59 \\
(57 \text { to } 61)\end{array}$ & $1 \cdot 2$ & $\begin{array}{l}81.9 \\
(73.9 \text { to } 89 \cdot 8)\end{array}$ & $\begin{array}{l}18 \cdot 3 \\
(18 \cdot 2 \text { to } 18 \cdot 5)\end{array}$ \\
\hline
\end{tabular}

$a$ Fibroblast inoculum $=0.56 \times 10^{\circ}$ per fiask

$b$ Glucose concentration in initial medium $=83 \mathrm{mg} . / 100 \mathrm{ml}$.

$c$ Leucocytes added (frozen-thawed): $10 \times 10^{\circ}$ per flask. 'Interaction' period 2 days

Table IX Effect of indomethacin, phenylbutazone, oxyphenbutazone, and hydrocortisone Na-succinate on fibroblast-leucocyte interaction

\begin{tabular}{|c|c|c|c|c|c|c|}
\hline $\begin{array}{l}\text { Fibroblast } \\
\text { strain }\end{array}$ & Additive & $\begin{array}{l}\text { Final cell } \\
\text { count/flask } \\
\times 10^{6}\end{array}$ & $\begin{array}{l}\text { Residual } \\
\text { medium } \\
\text { glucose } \\
(\mathrm{mg} . / 100 \mathrm{ml} .)\end{array}$ & $\begin{array}{l}\text { Glucose } \\
\text { uptake } \\
\text { (mg./flask } \\
\text { per } 2 \text { days) }\end{array}$ & $\begin{array}{l}\text { Total } \\
\text { HA/flask } \\
(\mu g .)\end{array}$ & $\begin{array}{l}\text { HA } \\
\text { synthesis rate } \\
\text { (pg./cell per day) }\end{array}$ \\
\hline VS-E & None & $\begin{array}{l}2 \cdot 67 \\
(2 \cdot 56 \text { to } 2 \cdot 78)\end{array}$ & $\begin{array}{l}43 \\
(42 \text { to } 44)\end{array}$ & $2 \cdot 3$ & $\begin{array}{l}238 \cdot 7 \\
(224 \cdot 5 \text { to } 253 \cdot 0)\end{array}$ & $\begin{array}{l}44 \cdot 7 \\
(44 \cdot 0 \text { to } 45 \cdot 5)\end{array}$ \\
\hline VS-E & Leucocytes $^{c}$ & $\begin{array}{l}1 \cdot 70 \\
(1 \cdot 67 \text { to } 1 \cdot 72)\end{array}$ & $\begin{array}{l}29 \\
(25 \text { to } 33)\end{array}$ & $3 \cdot 5$ & $\begin{array}{l}364 \cdot 2 \\
(364 \cdot 2 \text { to } 364 \cdot 2)\end{array}$ & $\begin{array}{l}106 \cdot 4 \\
(105 \cdot 9 \text { to } 109 \cdot 0)\end{array}$ \\
\hline VS-E & $\begin{array}{l}\text { Leucocytes }+ \\
\text { indomethacin } \\
(2 \mu \mathrm{g} . / \mathrm{ml} . \text { medium })\end{array}$ & $\begin{array}{l}3 \cdot 27 \\
(3 \cdot 24 \text { to } 3 \cdot 30)\end{array}$ & $\begin{array}{l}32 \\
(30 \text { to } 34)\end{array}$ & $2 \cdot 9$ & $\begin{array}{l}263 \cdot 7 \\
(255 \cdot 1 \text { to } 271 \cdot 4)\end{array}$ & $\begin{array}{l}40 \cdot 3 \\
(38 \cdot 6 \text { to } 41 \cdot 9)\end{array}$ \\
\hline$\overline{\text { VS-E }}$ & $\begin{array}{l}\text { Leucocytes }+ \\
\text { phenylbutazone } \\
(10 \mu \mathrm{g} . / \mathrm{ml} . \text { medium })\end{array}$ & $\begin{array}{l}2 \cdot 52 \\
(2 \cdot 40 \text { to } 2 \cdot 65)\end{array}$ & $\begin{array}{l}27 \\
(25 \text { to } 30)\end{array}$ & $3 \cdot 1$ & $\begin{array}{l}295 \cdot 6 \\
(273 \cdot 4 \text { to } 318 \cdot 4)\end{array}$ & $\begin{array}{l}58 \cdot 9 \\
(51 \cdot 6 \text { to } 66 \cdot 3)\end{array}$ \\
\hline VS-E & $\begin{array}{l}\text { Leucocytes }+ \\
\text { oxyphenbutazone } \\
(10 \mu \mathrm{g} . / \mathrm{ml} . \text { medium })\end{array}$ & $\begin{array}{l}2 \cdot 52 \\
(2 \cdot 50 \text { to } 2 \cdot 54)\end{array}$ & $\begin{array}{l}25 \\
(24 \text { to } 26)\end{array}$ & $3 \cdot 2$ & $\begin{array}{l}308 \cdot 1 \\
(308 \cdot 1 \text { to } 308 \cdot 1)\end{array}$ & $\begin{array}{l}61 \cdot 1 \\
(60 \cdot 6 \text { to } 61 \cdot 6)\end{array}$ \\
\hline VS-E & $\begin{array}{l}\text { Leucocytes }+ \\
\text { hydrocortisone } \\
(10 \mu \mathrm{g} . / \mathrm{ml} . \text { medium })\end{array}$ & $\begin{array}{l}2 \cdot 78 \\
(2 \cdot 46 \text { to } 3 \cdot 10)\end{array}$ & $\begin{array}{l}43 \cdot 5 \\
(42 \text { to } 45)\end{array}$ & $2 \cdot 3$ & $\begin{array}{l}242 \cdot 8 \\
(228 \cdot 6 \text { to } 257 \cdot 1)\end{array}$ & $\begin{array}{l}43 \cdot 9 \\
(41 \cdot 5 \text { to } 46 \cdot 4)\end{array}$ \\
\hline
\end{tabular}

$a$ Fibroblast inoculum $=0.56 \times 10^{\circ}$ per flask

$a$ Fibroblast inoculum $=0.56 \times 10^{\circ}$ per flask
$b$ Glucose concentration in initial medium $=90 \mathrm{mg} . / 100 \mathrm{ml}$

$c$ Leucocytes added (frozen-thawed): $10 \times 10^{\circ}$ per fiask. 'Interaction' period 2 davs 
affected joint by anti-inflammatory drugs is accompanied by a decrease in the pathological accumulation of hyaluronic acid in the joint fluid and an increase in the glucose concentration of the synovial fluid. The anti-inflammatory drugs used in our experiments imitated in some respects their effect in vivo. The interaction of fibroblasts and leucocytes in culture offers a useful model for the investigation of the inflammatory process.

\section{Summary}

Frozen-thawed leucocytes were added to cultured monolayers of human synovial fibroblasts. A 23 to 140 per cent. increase in hyaluronic acid synthesis (expressed per fibroblast) and a 5 to 100 per cent. increase in glucose consumption (per flask) were found 1 to 2 days after the addition of the leucocytes. This stimulation was inhibited by adding antiinflammatory drugs with the leucocytes:

Hydrocortisone acetate $1 \mu \mathrm{g} . / \mathrm{ml}$. (40 and 100 per cent. inhibition of hyaluronate overproduction in two experiments);

Hydrocortisone Na-succinate $1 \mu \mathrm{g} . / \mathrm{ml}$. (100 per cent. in two experiments);

Indomethacin 2 or $4 \mu \mathrm{g} . / \mathrm{ml}$. (100 per cent. in three experiments);

Phenylbutazone 10 or $20 \mu \mathrm{g} . / \mathrm{ml}$. (75 and 100 per cent.);

Sodium salicylate $200 \mu \mathrm{g} . / \mathrm{ml}$. (44 and 88 per cent.).

The inhibition of hyaluronate synthesis was associated with a 5 to 47 per cent. decrease in glucose uptake and no toxic effect on the fibroblasts was noted. The effect of anti-inflammatory drugs in vitro duplicated, in some respects, their action in vivo. The interaction of fibroblasts and leucocytes in culture offers a useful model for the investigation of the inflammatory process.

We wish to express our gratitude to the Department of Orthopaedic Surgery, Beilinson Hospital, for providing the synovial tissue material and to Mrs. Dozia Federman for technical assistance.

\section{References}

CASTOR, C. W. (1962) J. Lab. clin. Med., 60, 788 (Adrenocorticoid suppression of mucopolysaccharide formation in human connective tissue cell cultures).

-, Dorstewitz, E. L., Wright, D., AND RrTCHIE, J. C. (1969) Ibid., 74, 860 (Connective tissue regulation: the effect of a polypeptide factor on connective tissue metabolism).

-, Prince, R. K., AND Dorstewrtz, E. L. (1962) Lab. Invest., 11, 703 (Characteristics of human 'fibroblasts' cultivated in vitro from different anatomical sites).

-, Wright, D., AND BuckinghaM, R. B. (1968) Arthr. and Rheum., 11, 652 (Effects of rheumatoid sera on fibroblast proliferation and hyaluronic acid synthesis).

DelaunaY, A., AND Basin, S. (1965) 'Non-steroidal Anti-inflammatory Drugs'. Proceedings of an International Symposium, ed. S. Garattini and M. N. G. Dukes, p. 25. Int. Congr. Ser., No. 82. Excerpta Medica Foundation, Amsterdam (Metabolic changes in connective tissue during an inflammatory process).

Kalbhen, D. A., KARZeL, K., AND DomenJoz, R. (1967) Med. Pharmacol. exp. (Basel), 16, 185 (The inhibitory effects of some antiphlogistic drugs on the glucosamine incorporation into mucopolysaccharide synthesized by fibroblast cultures).

Kingsley, G. R., AND Getchell, G. (1960) Clin. Chem., 6, 466 (Direct ultramicro glucose oxidase method for determination of glucose in biological fluids).

YARON, M., AND CASTOR, C. W. (1969) Arthr. and Rheum., 12, 365 (Leukocyte-connective tissue cell interaction. I. Stimulation of hyaluronate synthesis by live and dead leukocytes). 